\title{
Bifurcações de Equilíbrio de Fronteira no Controle de um Conversor Bidirecional Conectado a uma Microrede CC
}

\author{
Daniel J. Pagano, Rony Cristiano \\ Depto de Automação e Sistemas, DAS, UFSC \\ Campus Trindade \\ 88040-900, Florianópolis, SC \\ E-mail: daniel.pagano@ufsc.br, rony.cristiano@ufsc.br
}

Resumo: As microredes cc são uma opção para novas demandas por qualidade de energia $e$ integração de fontes renováveis no sistema elétrico. Numa microrede em modo ilhado, o conversor bidirecional cc-cc (DBC) tem o papel de controlar o balanço de potência entre geração e demanda de carga, utilizando a bateria para drenar ou injetar potência na rede. O conversor bidirecional com controle por modos deslizantes (SMC) é modelado como um sistema dinâmico DPWS. Esta classe de sistemas exibe bifurcações típicas, denominadas bifurcações induzidas pela descontinuidade (DIBs), como por exemplo a Bifurcação do Equilíbrio de Fronteira (BEBs), que é foco de estudo deste trabalho.

Palavras-chave: Microrede CC, conversor bidirecional, sistemas dinâmicos DPWS, controle por modos deslizantes, bifurcações do equilíbrio de fronteira.

\section{Introdução}

Microredes (MRs) são sistemas de distribuição de energia de corrente alternada (ca) ou corrente contínua (cc) compostos por células solares fotovoltaicas (PV), geradores eólicos, células de combustível e baterias. Em destaque, as MRs cc são uma opção para novas demandas por qualidade de energia e integração de fontes renováveis no sistema elétrico [6].

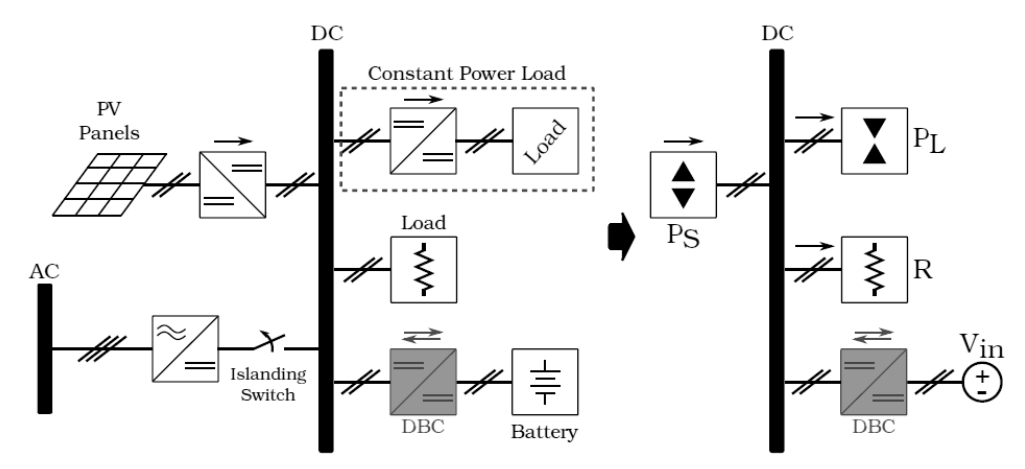

Figura 1: Modelo de uma Microrrede CC operando em modo ilhado.

Na dinâmica da MR cc, conversores no ponto de carga são modelados como Constant Power Loads-CPLs $\left(P_{L}\right)$ e cargas diretamente conectadas ao barramento como cargas resistivas $(R)$. As fontes (solar, eólica, etc.) conectadas ao barramento cc, são modeladas como fontes de potência $\left(P_{S}\right)$ e a bateria é considerada uma fonte de tensão cc ideal $\left(V_{i n}\right)$. A MR no modo ilhado e o seu modelo equivalente estão ilustrados na Figura 1. 
A potência gerada e a consumida pela CPL podem ser modeladas como $P=P_{S}+P_{L}$, resultando no modelo simplificado ilustrado na Figura 2. Assim, o conversor bidirecional (DBC) tem o papel de controlar o balanço de potência entre geração e demanda de carga, utilizando a bateria para drenar ou injetar potência na rede.

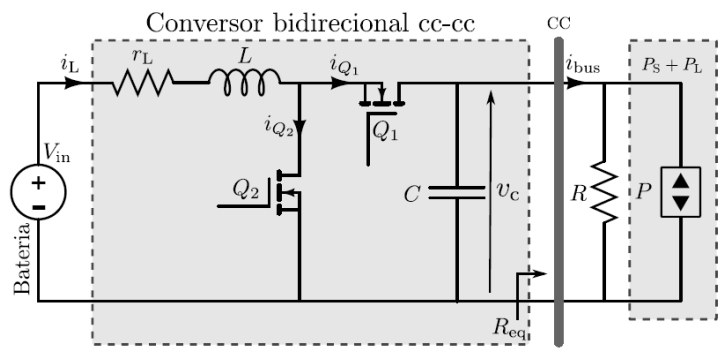

Figura 2: Modelo simplificado de uma Microrrede CC operando em modo ilhado.

Os conversores de potência são modelados matematicamente por sistemas dinâmicos DPWS (Discontinuous Piece-Wise Smooth), na literatura conhecidos como Sistemas de Filippov [4]. O correspondente modelo matemático é um conjunto de equações diferenciais ordinárias, caracterizado por algumas descontinuidades no próprio campo vetorial. Estas descontinuidades aparecem apenas em um pequeno subconjunto do espaço de estados e geralmente tal subconjunto é uma variedade suave genericamente transversal ao campo vetorial. Esta classe de sistemas pode apresentar órbitas deslizantes que são confinadas à superfície de comutação.

Sistemas dinâmicos DPWS exibem uma nova classe de bifurcações, denominadas bifurcações induzidas pela descontinuidade (DIBs, Discontinuity Induced Bifurcations), pois são sistemas de natureza descontínua [2]. Um exemplo é a Bifurcação do Equilíbrio de Fronteira (BEBs, Boundary Equilibrium Bifurcations) onde, sob variação de parâmetros, é observado uma colisão de um ponto de equilíbrio com a superfície de comutação [3].

Neste trabalho, aplica-se o controle por modos deslizantes (SMC) com filtro Washout ao conversor boost bidirecional conectado numa bateria para regular a tensão em uma Microrede cc em modo ilhado, com o objetivo de identificar a ocorrência das BEBs. Do ponto de vista de controle, o estudo das BEBs se faz necessário pois, mesmo garantindo estabilidade do ponto de equilíbrio, uma perturbação de carga pode levar este ponto a desaparecer, transformando-o num equilíbrio virtual. Na seção (2) é apresentado algumas das propriedades dos sistemas dinâmicos DPWS, defini-se os tipos de equilíbrios, as características e classificação das BEBs. Na seção (3), a partir do sistema normalizado do conversor boost bidirecional, defini-se a estratégia de controle e faz-se um estudo dos equilíbrios para identificar e comprovar a ocorrência das BEBs.

\section{Sistemas dinâmicos DPWS e as BEBs}

Suponha que são dados dois campos vetoriais suaves $\mathbf{F}^{ \pm}: \mathbb{R}^{n} \rightarrow \mathbb{R}^{n}$ e a função escalar $h: \mathbb{R}^{n} \rightarrow \mathbb{R}$, tal que a variedade de comutação $\Sigma=\left\{\mathbf{x} \in \mathbb{R}^{n}, h(\mathbf{x})=0\right\}$ é uma superfície suave, com gradiente de $h$ diferente de zero. Em seguida, define-se as duas regiões abertas $R^{-}=\left\{\mathbf{x} \in \mathbb{R}^{n}: h(\mathbf{x})<0\right\}$ e $R^{+}=\left\{\mathbf{x} \in \mathbb{R}^{n}: h(\mathbf{x})>0\right\}$, e o sistema dinâmico DPWS

$$
\dot{\mathbf{x}}= \begin{cases}\mathbf{F}^{-}(\mathbf{x}, \mu), & \text { se } \mathbf{x} \in R^{-} \\ \mathbf{F}^{+}(\mathbf{x}, \mu), & \text { se } \mathbf{x} \in R^{+} .\end{cases}
$$

dependente do parâmetro $\mu \in \mathbb{R}$.

A superfície de comutação $\Sigma$ pode ser particionada em três diferentes regiões: uma onde $\mathbf{F}^{ \pm}$ apontam para superfície em ambos os lados, chamada região de deslizamento atrativo $\Sigma_{a s}$; outra 
onde $\mathbf{F}^{ \pm}$apontam para fora da superfície em ambos os lados, chamada região de deslizamento repulsivo $\Sigma_{r s}$; e outra onde um dos campos vetoriais aponta para superfície e o outro aponta para fora, chamada região de cruzamento $\Sigma_{c}$.

No conjunto deslizante, dado pela união $\Sigma_{a s} \cup \Sigma_{r s}$, a dinâmica é descrita pelo campo vetorial deslizante calculado a partir de uma combinação dos campos vetoriais originais [4], da forma,

$$
\mathbf{F}_{s}(\mathbf{x}, \mu)=(1-\lambda) \mathbf{F}^{-}(\mathbf{x}, \mu)+\lambda \mathbf{F}^{+}(\mathbf{x}, \mu) .
$$

Para cada $\mathbf{x} \in \Sigma_{a s} \cup \Sigma_{r s}$, o valor de $\lambda$ é selecionado tal que $h_{\mathbf{x}} \cdot \mathbf{F}_{s}=0$ ( $h_{\mathbf{x}}$ é o gradiente de $h$ ), ou seja,

$$
\lambda=\lambda(\mathbf{x}, \mu)=\frac{h_{\mathbf{x}} \cdot \mathbf{F}^{-}(\mathbf{x}, \mu)}{h_{\mathbf{x}} \cdot\left(\mathbf{F}^{-}(\mathbf{x}, \mu)-\mathbf{F}^{+}(\mathbf{x}, \mu)\right)},
$$

desde que o denominador acima seja diferente de zero. Então, $\lambda \in(0,1) \forall \mathbf{x} \in \Sigma_{a s} \cup \Sigma_{r s}$.

O sistema dinâmico DPWS (1) possui os equilíbrios de cada campo vetorial $\mathbf{F}^{ \pm}$e também os equilíbrios do campo vetorial deslizante $\mathbf{F}_{s}$, mas é importante distinguir entre equilíbrios reais ou virtuais. Segue as definições:

Definição 2.1 Seja $\overline{\mathbf{x}} \in \mathbb{R}^{n}$, dependente ou não do parâmetro $\mu$, um ponto de equilíbrio do campo vetorial $\mathbf{F}^{-}\left(\mathbf{F}^{+}\right)$, isto é, $\mathbf{F}^{-}(\overline{\mathbf{x}}, \mu)=\mathbf{0}\left(\mathbf{F}^{+}(\overline{\mathbf{x}}, \mu)=\mathbf{0}\right)$. Este ponto é um equilíbrio real de $\mathbf{F}^{-}$se $h(\overline{\mathbf{x}})<0(h(\overline{\mathbf{x}})>0)$; ou um equilíbrio virtual se $h(\overline{\mathbf{x}})>0(h(\overline{\mathbf{x}})<0)$; ou um equilíbrio de fronteira se $h(\overline{\mathbf{x}})=0$.

Definição 2.2 Um ponto $\tilde{\mathbf{x}} \in \mathbb{R}^{n}$, dependente ou não do parâmetro $\mu$, é um pseudo-equilíbrio do sistema dinâmico DPWS (1) se $\tilde{\mathbf{x}} \in \Sigma$ e é um ponto de equilíbrio do campo vetorial deslizante $\mathbf{F}_{s}$ dado em (2), ou seja,

$$
\begin{aligned}
(1-\lambda) \mathbf{F}^{-}(\tilde{\mathbf{x}}, \mu)+\lambda \mathbf{F}^{+}(\tilde{\mathbf{x}}, \mu) & =\mathbf{0} \\
h(\tilde{\mathbf{x}}) & =0
\end{aligned}
$$

Além disso, $\tilde{\mathbf{x}}$ é um pseudo-equilíbrio real quando $\lambda \in(0,1)$, e virtual quando $\lambda<0$ ou $\lambda>1$.

O equilíbrio de fronteira é um ponto crítico de mudança entre equilíbrio real e virtual. Esta mudança topológica de equilíbrio real para virtual (ou vice-versa) é influenciada pela variação do parâmetro $\mu$, e caracteriza uma Bifurcação do Equilíbrio de Fronteira (BEB).

Suponha que no valor crítico $\mu=0$, o campo vetorial $\mathbf{F}^{-}$possui um ponto de equilíbrio de fronteira. A BEB ocorre de duas formas: uma denominada Persistência, ocorre quando em $\mu<0$ o equilíbrio de $\mathbf{F}^{-}$é real e o pseudo-equilíbrio é virtual, para $\mu=0$ eles colidem formando o equilíbrio de fronteira, e para $\mu>0$ o equilíbrio torna-se virtual e o pseudo-equilíbrio tornase real. A outra, denominada Dobra não Suave, ocorre quando em $\mu<0$ o equilíbrio e o pseudo-equilíbrio são reais, para $\mu=0$ eles colidem, e para $\mu>0$ ambos tornam-se virtuais [3]. Note que, no caso da Persistência o equilíbrio do campo $\mathbf{F}^{-}$e o equilíbrio do campo deslizante $\mathbf{F}_{s}$ (pseudo-equilíbrio) não coexistem para $\mu \neq 0$, e no caso da Dobra não suave eles podem coexistir para $\mu<0$ (ou $\mu>0)$.

\section{Estudo das BEBs no Controle do Conversor Bidirecional}

Para o estudo das BEBs, considera-se o modelo do conversor boost bidirecional, mostrado na Figura 2, na forma normalizada como em [6], dada por

$$
\begin{aligned}
& \dot{x}=1-b x-u y \\
& \dot{y}=u x-a y-\frac{d}{y},
\end{aligned}
$$


em que $u \in\{0,1\}$ é a ação de controle, $a \in \mathbb{R}_{+}, b \in \mathbb{R}_{+}$e $d \in \mathbb{R}$ são parâmetros, e $x \in \mathbb{R}$ e $y \in \mathbb{R}^{+}-\{0\}$ as variáveis de estados do sistema.

Os parâmetros $a$ e $d$ representam as cargas resistiva e de potência normalizadas do conversor. Estes parâmetros variam com a demanda de potência e com a disponibilidade de fontes da MR, gerando consequentemente mudanças na dinâmica do sistema. Para superar este problema, adota-se uma estratégia de controle SMC com filtro high-pass denominado Washout. Este filtro, juntamente com uma escolha apropriada da superfície de comutação, eliminam a dependência do ponto de operação de interesse em relação aos parâmetros $a$ e $d$ [1].

O filtro Washout introduz uma nova variável ao sistema, com equação dinâmica $\dot{z}=\omega(x-z)$, e $\omega>0$. No equilíbrio, $x$ e $z$ são iguais, assim $(x-z)$ trabalha como uma medida do erro que alimenta o controlador SMC apenas durante o transitório. Em seguida, define-se uma estratégia de controle por modos deslizantes baseada na ação de controle $u=\frac{1}{2}(1+\operatorname{sign}[h(\mathbf{x})])$ com $\mathbf{x}=(x, y, z) \in \mathbb{R}^{3}$, onde $h: \mathbb{R}^{3} \rightarrow \mathbb{R}$ é uma função escalar da forma

$$
h(\mathbf{x})=y-y_{r}+k(x-z)
$$

com $k>0$ (parâmetro real de controle) e $y_{r}>1$ (tensão normalizada de referência). Assim, representa-se a superfície de comutação pelo conjunto $\Sigma=\left\{\mathbf{x} \in \mathbb{R}^{3}: h(\mathbf{x})=0\right\}$.

O sistema de controle em questão pode ser representado como um sistema dinâmico DPWS

$$
\dot{\mathbf{x}}=\left\{\begin{array}{lll}
\mathbf{F}^{-}(\mathbf{x}, d), & \text { se } & h(\mathbf{x})<0 \\
\mathbf{F}^{+}(\mathbf{x}, d), & \text { se } & h(\mathbf{x})>0
\end{array},\right.
$$

com os campos vetoriais

$$
\mathbf{F}^{-}(\mathbf{x}, d)=\left[\begin{array}{c}
1-b x \\
-a y-\frac{d}{y} \\
\omega(x-z)
\end{array}\right] \quad \text { e } \mathbf{F}^{+}(\mathbf{x}, d)=\left[\begin{array}{c}
1-b x-y \\
x-a y-\frac{d}{y} \\
\omega(x-z)
\end{array}\right],
$$

dependentes do parâmetro $d \in \mathbb{R}$. Como $y>0, \mathbf{F}^{-}$possui apenas um ponto de equilíbrio: $\overline{\mathbf{x}}^{-}(d)=\left(\frac{1}{b}, \sqrt{\frac{-d}{a}}, \frac{1}{b}\right)$, definido para $d \leq 0$. O campo $\mathbf{F}^{+}$possui dois pontos de equilíbrio: $\overline{\mathbf{x}}_{1}^{+}(d)=\left(\bar{x}_{1}, \bar{y}_{1}, \bar{x}_{1}\right)$ definido para $0<d \leq d_{S N}$, e $\overline{\mathbf{x}}_{2}^{+}(d)=\left(\bar{x}_{2}, \bar{y}_{2}, \bar{x}_{2}\right)$ para $d \leq d_{S N}$; com

$$
\bar{x}_{1,2}=\frac{2(a+d)}{1+2 a b \mp \sqrt{1-d / d_{S N}}}, \quad \bar{y}_{1,2}=\frac{1 \mp \sqrt{1-d / d_{S N}}}{2(1+a b)}
$$

$\mathrm{e}$

$$
d_{S N}=\frac{1}{4 b(1+a b)}
$$

Seguindo a definição (2.2), para determinar os pontos de pseudo-equilíbrio resolve-se o sistema de equações (4)-(5), em termos de $(\mathbf{x}, \lambda)$, obtendo $\left(\tilde{\mathbf{x}}_{+}(d), \tilde{\lambda}_{-}(d)\right)$ e $\left(\tilde{\mathbf{x}}_{-}(d), \tilde{\lambda}_{+}(d)\right)$ com

$$
\begin{aligned}
& \tilde{\mathbf{x}}_{ \pm}(d)=\left(\tilde{x}_{ \pm}, y_{r}, \tilde{x}_{ \pm}\right), \text {em que } \tilde{x}_{ \pm}=\frac{1 \pm \sqrt{1-4 b\left(d+a y_{r}^{2}\right)}}{2 b} \\
& \tilde{\lambda}_{\mp}(d)=\frac{1 \mp \sqrt{1-4 b\left(d+a y_{r}^{2}\right)}}{2 y_{r}}
\end{aligned}
$$

para $d<d_{S N}^{s}$ com

$$
d_{S N}^{s}=\frac{1-4 a b y_{r}^{2}}{4 b}
$$

Para identificar a ocorrência da BEB, considere primeiro uma análise no campo vetorial $\mathbf{F}^{-}$ e depois no campo vetorial $\mathbf{F}^{+}$. O equilíbrio do campo vetorial $\mathbf{F}^{-}$é o ponto $\overline{\mathbf{x}}^{-}(d)$, cuja posição no espaço de estados depende do parâmetro $d$. A trajetória descrita por este ponto é uma curva 
suave do $\mathbb{R}^{3}$ definida para $d \in \mathbb{R}^{+}$, que cruza transversalmente a superfície de comutação $\Sigma$ no ponto $\overline{\mathbf{x}}^{-}\left(d_{B E B^{-}}\right)=\left(1 / b, y_{r}, 1 / b\right)$ quando $d$ assume o valor crítico

$$
d=d_{B E B^{-}}=-a y_{r}^{2},
$$

pois, $h\left(\overline{\mathbf{x}}^{-}\left(d_{B E B^{-}}\right)\right)=0$ e $h^{\prime}\left(\overline{\mathbf{x}}^{-}\left(d_{B E B^{-}}\right)\right)=-\frac{1}{2 a y_{r}}<0$. A derivada negativa indica que $\overline{\mathbf{x}}^{-}(d)$ é um equilíbrio virtual para $d<d_{B E B^{-}}$, e real para $d>d_{B E B^{-}}$( $d$ suficientemente próximo à $\left.d_{B E B^{-}}\right)$. No valor crítico $d=d_{B E B^{-}}$, o equilíbrio $\overline{\mathbf{x}}^{-}(d)$ colide com o pseudo-equilíbrio $\tilde{\mathbf{x}}_{+}(d)$, formando o equilíbrio de fronteira. Este é um ponto sobre a linha limite das regiões de deslizamento atrativo/repulsivo e cruzamento, pois, $\tilde{\lambda}_{-}\left(d_{B E B^{-}}\right)=0$. O pseudo-equilíbrio $\tilde{\mathbf{x}}_{+}(d)$ cruza esta linha limite de forma transversal, pois $\tilde{\lambda}_{-}^{\prime}\left(d_{B E B^{-}}\right)=b / y_{r}>0$. A derivada positiva indica que $\tilde{\mathbf{x}}_{+}(d)$ é um pseudo-equilíbrio virtual para $d<d_{B E B^{-}}$, e real para $d>d_{B E B^{-}}$. Então, quando $d=d_{B E B^{-}}$ocorre uma BEB do tipo Dobra não Suave (Nonsmooth fold). Na figura 3(a) a curva de cor roxa, com equação dada em (15), indica esta bifurcação no plano $\left(y_{r}, d\right)$.

No campo vetorial $\mathbf{F}^{+}$o equilíbrio $\overline{\mathbf{x}}_{1}^{+}(d)$ é sempre virtual, pois $y_{r}>1$. O outro equilíbrio, $\overline{\mathbf{x}}_{2}^{+}(d)$, descreve uma curva suave no $\mathbb{R}^{3}$ que cruza transversalmente a superfície de comutação $\Sigma$ no ponto $\overline{\mathbf{x}}_{2}^{+}\left(d_{B E B^{+}}\right)=\left(\frac{1-y_{r}}{b}, y_{r}, \frac{1-y_{r}}{b}\right)$ quando $d$ assume o valor crítico

$$
d=d_{B E B^{+}}=\frac{y_{r}-(1+a b) y_{r}^{2}}{b},
$$

pois, $h\left(\overline{\mathbf{x}}_{2}^{+}\left(d_{B E B^{+}}\right)\right)=0$ e $h^{\prime}\left(\overline{\mathbf{x}}_{2}^{+}\left(d_{B E B^{+}}\right)\right)=-\frac{b}{\left|2 y_{r}(1+a b)-1\right|}<0$. A derivada negativa indica que $\overline{\mathbf{x}}_{2}^{+}(d)$ é um equilíbrio real para $d<d_{B E B^{+}}$e virtual para $d>d_{B E B^{+}}$( $d$ suficientemente próximo à $\left.d_{B E B^{+}}\right)$. No valor crítico $d=d_{B E B^{+}}, \overline{\mathbf{x}}_{2}^{+}(d)$ colide com o pseudo-equilíbrio $\tilde{\mathbf{x}}_{-}(d)$ formando o equilíbrio de fronteira. Este é um ponto sobre a linha limite das regiões de deslizamento atrativo/repulsivo e cruzamento, pois, $\tilde{\lambda}_{+}\left(d_{B E B^{+}}\right)=1$. O pseudo-equilíbrio $\tilde{\mathbf{x}}_{-}(d)$ cruza esta linha limite de forma transversal, pois, $\tilde{\lambda}_{+}^{\prime}\left(d_{B E B^{+}}\right)=-\frac{b}{y_{r}\left|2 y_{r}-1\right|}<0$. A derivada negativa indica que $\tilde{\mathbf{x}}_{-}(d)$ é um pseudo-equilíbrio virtual para $d<d_{B E B^{+}}$e real para $d>d_{B E B^{+}}$. Então, no valor crítico $d=d_{B E B^{+}}$ocorre uma BEB do tipo Persistência (Persistence). Na figura 3(a) a curva de cor azul, com equação dada em (16), indica esta bifurcação no plano $\left(y_{r}, d\right)$.

As conclusões obtidas sobre as BEBs são válidas apenas para $y_{r}>1$, característica do conversor boost. No entanto, supondo $y_{r}=1 / 2$, quando $d=d_{B E B^{+}}$no ponto de equilíbrio de fronteira ocorre uma tripla colisão, envolvendo os equilíbrios $\overline{\mathbf{x}}_{2}^{+}$e $\tilde{\mathbf{x}}_{ \pm}$. Neste caso, o equilíbrio de fronteira é um ponto GBE [5], Generalized Boundary Equilibrium. Esta situação degenerada é uma bifurcação de codimensão 2, que separa os dois cenários: Persistência e Dobra não suave.

Na Figura 3 é mostrado um plano de bifurcações das BEBs, e bifurcações Sela-Nó $\left(S N_{1}\right.$ em $\mathbf{F}^{+}$, linha verde; e $S N_{2}$ em $\mathbf{F}_{s}$, linha vermelha; de equações dadas em (11) e (14).). Também, resultados de simulação do sistema dinâmico (9), considerando $b=0.06, \omega=0.6, y_{r}=1.2, k=1$, $a=0.3$ e $d \in\{-5,-4.432,-4\}$; onde é visualizada a ocorrência da BEB do tipo Persistência.

\section{Conclusões}

Neste trabalho foram abordadas algumas das propriedades de sistemas dinâmicos DPWS como, o movimento deslizante, pseudo-equilíbrio, equilíbrio de fronteira, além da caracterização das BEBs e da definição dos dois tipos: Persistência e Dobra não Suave. Com o objetivo de identificar e comprovar a ocorrência das BEBs em sistemas reais, foi considerado uma aplicação ligada ao controle de um conversor Boost Bidirecional conectado a uma bateria em uma Microrede cc quando em modo ilhado. Para o processo de controle foi utilizado um SMC introduzindo o filtro Washout, responsável por manter o ponto de operação de interesse após uma perturbação de carga. Os resultados obtidos neste trabalho comprovam a ocorrência das BEBs no sistema de controle proposto. Este fato é de extrema importância, pois uma perturbação de carga pode instabilizar o sistema ou mudar o ponto de operação, sendo que neste caso o filtro Washout é ineficiente para colocar o sistema de volta ao ponto de operação de interesse. 


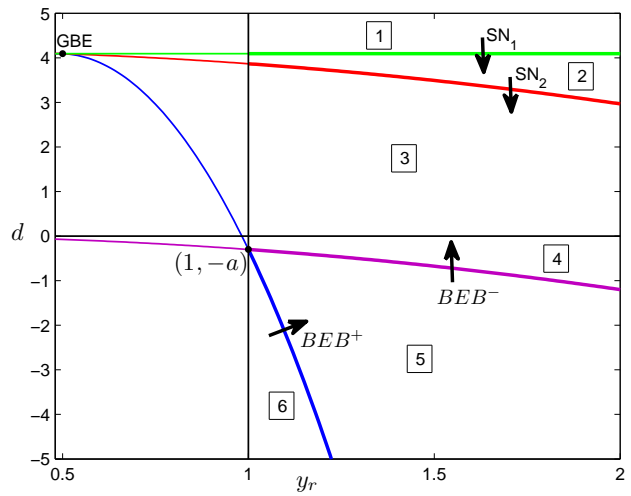

(a) Plano $\left(y_{r}, d\right)$ de bifurcações.

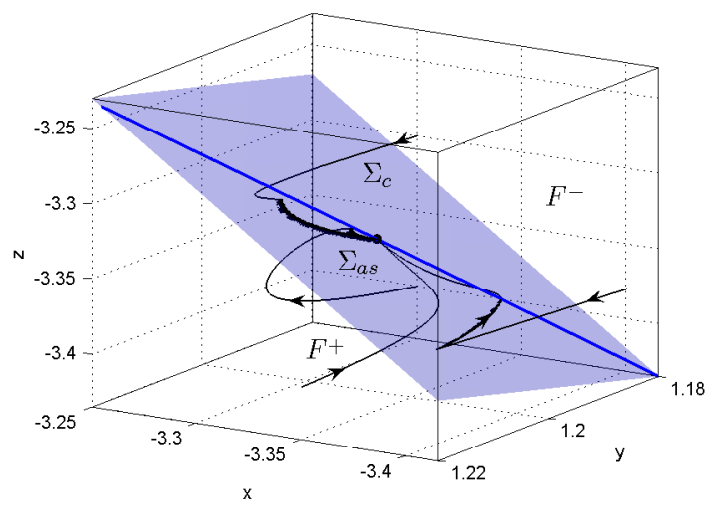

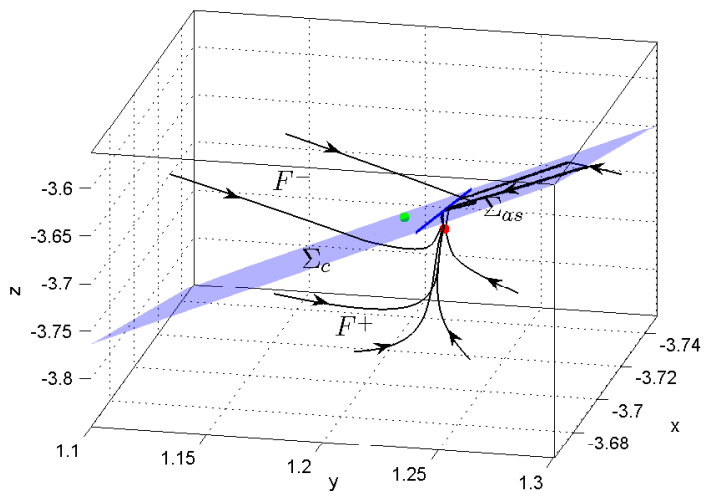

(b) Região $6, d=-5<d_{B E B^{+}}: \overline{\mathbf{x}}_{2}^{+}$(ponto vermelho) real e $\tilde{\mathbf{x}}_{-}$(ponto verde) virtual.

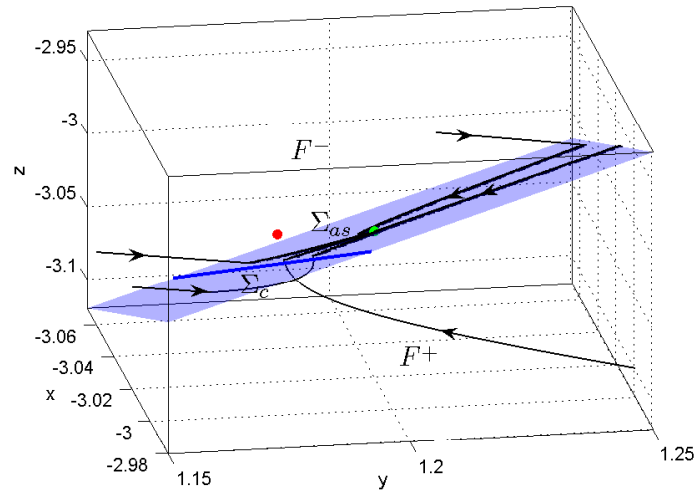

(c) Sobre a curva de bifurcação $\mathrm{BEB}^{+}, d=-4.432=(\mathrm{d})$ Região $5, d=-4>d_{B E B^{+}}$: $\overline{\mathbf{x}}_{2}^{+}$virtual e $\tilde{\mathbf{x}}_{-}$real. $d_{B E B^{+}}$: equilíbrio de fronteira.

Figura 3: Conjunto de bifurcações e resultados de simulação.

\section{Referências}

[1] R. Cristiano. Bifurcações em Sistemas Dinâmicos DPWS com Aplicações em Eletrônica de Potência. Dissertação de mestrado. PGEAS, UFSC. Em andamento, previsão, Outubro de 2013. Orientador: Daniel J. Pagano.

[2] M. di Bernardo, A. Nordmark and G. Olivar, (2008b). Discontinuity-induced bifurcations of equilibria in piecewise-smooth dynamical systems; Physica D 237, 119-136.

[3] M. di Bernardo, D. J. Pagano and E. Ponce. Nonhyperbolic boundary equilibrium bifurcations in planar Filippov systems: A case study approach. Int. J. Bifurcat. Chaos, vol. 18, pp. 1377-1392, 2008.

[4] A. F. Filippov. Differential Equations with Discontinuous Righthand Sides. Dordrecht, The Netherlands: Kluwer Academic, 1988.

[5] F. D. Rossa and F. Dercole. Generic and generalized boundary operating points in piecewiselinear (discontinuous) control systems; in 51st IEEE Conference on Decision and Control (accepted), 2012.

[6] A. P. N. Tahim, D. J. Pagano e E. Ponce. Nonlinear Control of Boost Bidirectional Converters in Stand-alone dc Microgrids. 51st IEEE Conference on Decision and Control - CDC. Maui, Hawaii, USA; 2012. 